\title{
THE "OVERCONVERGENCE" OF BOUNDED NORMAL FAMILIES OF ANALYTIC FUNCTIONS
}

\section{ALAN WILSON}

Introduction. Let the power series $f(z)=\sum_{\nu=0}^{\infty} a_{\nu} z^{\nu}$ have radius of convergence 1. A subsequence of the partial sums of $f(z)$ is said to be overconvergent if it converges to $f(z)$ uniformly in the neighborhood of a regular point of $f(z)$ on $|z|=1$. We shall formulate and prove direct generalizations of the two theorems of Ostrowski which provide necessary [1] and sufficient [2] conditions for the overconvergence of a power series.

Ostrowski's theorems represent a generalization of the Hadamard gap theorem [3;4]. Recently, Johnson [5] extended the Hadamard gap theorem to locally bounded families of analytic functions. Our results (Theorems 1 and 2) relate to Johnson's theorem as Ostrowski's results relate to Hadamard's theorem. The methods we use are, in Theorem 1, Mordell's [6] and, in Theorem 2, Ostrowski's.

Definition 1 describes an extended form of uniform convergence which Lemma 1 shows to be useful in describing the behavior of certain subfamilies of the family of partial sums of a bounded family of power series. Definition 2 presents a generalization of overconvergence which Lemma 2 shows to be, in fact, an overconvergence for bounded families of power series.

Notation. $\mathcal{F}=\{f(z)\}=\left\{\sum_{\nu=0}^{\infty} a_{\nu}(f) z^{\nu}\right\}$ shall denote a family of functions with positive radius of regularity at the origin (see [5]) which, in addition, is locally bounded in $|z|<R$. That is, $|z|<R$ is the largest circle about the origin wherein every $f$ is holomorphic and $F$ is a normal family whose closure does not contain the constant $\infty$. For brevity we shall call $R$ the radius of finite normality of $\mathcal{F}$ (at the origin). Therefore [5], each of the numbers

$$
\sup _{\mathcal{F}}\left|a_{\nu}(f)\right|(\nu=0,1,2, \cdots)
$$

is finite and

$$
\varlimsup_{\nu \rightarrow \infty} \sup _{\mathcal{F}}\left|a_{\nu}(f)\right|^{1 / \nu}=1 / R .
$$

In our terms, ${ }^{1}$ a point $z_{0}$ of $|z|=R$ is a regular point of $\mathcal{F}$ if there is a positive number $r$ such that (a) every $f$ can be continued directly into $\left|z-z_{0}\right|<r$ and (b) the resulting family of continuations is locally

Received by the editors May 3, 1960.

${ }^{1}$ For a more general definition of regular point see [5]. 
bounded in $\left|z-z_{0}\right|<r$. If no point of $|z|=\Re$ is a regular point, $|z|=R$ is a $c u t$ for $\mathfrak{F}$.

We put $\sum_{\nu=0}^{n} a_{\nu}(f) z^{\nu}=S_{n}(f, z)$ and call $\Sigma=\left\{S_{n}(f, z)\right\} n \geqq 0, f \in \mathcal{F}$ the complete family of partial sums of $\mathcal{F}$. By a subfamily of $\Sigma$ we shall always mean a subset of $\Sigma$ determined by a family $\left\{m_{n}(f)\right\} n \geqq 1, f \in \mathcal{F}$ of sequences of integers, where, for every $f, 0 \leqq m_{1}(f)<m_{2}(f)<\cdots$, $m_{n}(f) \rightarrow \infty$. We denote $\left\{S_{m_{n}(f)}(f, z)\right\}$ by $\Sigma\left[m_{n}(f)\right]$. In particular, $\Sigma[n]=\Sigma$.

Definition 1. The subfamily of partial sums $\Sigma\left[m_{n}(f)\right]$ converges uniformly to $\mathcal{F}$ on a set $\Delta$ if, given $\epsilon>0$, there is an $N(\epsilon, \Delta)=N$, independent of the functions $f(z)$, such that

$$
\left|S_{m_{n}(f)}(f, z)-f(z)\right|<\epsilon, \quad \text { for } n>N, f \in \mathcal{F}, z \in \Delta .
$$

LeMma 1. If $\mathcal{F}$ has (positive) radius of finite normality $R$, then every subfamily of partial sums $\Sigma\left[m_{n}(f)\right]$ converges uniformly to $\mathcal{F}$ on every compact subset of $|z|<R$.

Proof. The functions $f(z)-S_{m_{n}(f)}(f, z)$ are the remainders of power series and are easily shown to be uniformly small (with respect to $\mathscr{F}$ ) on every closed circle $|z| \leqq r<R$. One uses the formula (1) for $R$ and the fact that $m_{n}(f) \rightarrow \infty$ for every $f$.

DeFinition 2. Let $\mathcal{F}$ have radius of finite normality $R(0<R<\infty)$. We say that a subfamily $\Sigma\left[m_{n}(f)\right]$ of the partial sums of $\mathcal{F}$ is overconvergent if (a) $|z|=R$ is not a cut for $\mathcal{F}$, and (b) there is a simply connected domain $D$, containing $|z|<R$ and the points of $|z|=R$ where $\mathcal{F}$ is regular, such that $\Sigma\left[m_{n}(f)\right]$ converges uniformly to $\mathcal{F}$ on every compact subset of $D$.

LemMa 2. Let $\mathcal{F}$ have radius of finite normality $R(0<R<\infty)$. Then the complete family of partial sums of $\mathcal{F}$ is never overconvergent.

Proof. The assertion is trivial if $|z|=\mathscr{R}$ is a cut. Suppose that $|z|=R$ is not a cut and that $\Sigma[n]$ is overconvergent. By Definition 2 there is a point $z_{0},\left|z_{0}\right|>\Re$, where $\mathcal{F}$ is uniformly bounded $\left(\left|f\left(z_{0}\right)\right|\right.$ $\leqq M, f \in \mathcal{F})$. By Definition 1 there is an $N\left(1, z_{0}\right)=N$ such that $\left|S_{n}\left(f, z_{0}\right)\right| \leqq M+1$ for $n>N, f \in \mathcal{F}$. Therefore, for $n>N$ and for all $f$

$$
\begin{aligned}
\left|a_{n}(f) z_{0}\right| & =\left|S_{n}\left(f, z_{0}\right)-S_{n-1}\left(f, z_{0}\right)\right| \\
& \leqq 2(M+1), \\
\sup _{\mathcal{F}}\left|a_{n}(f)\right| 1 / n\left|z_{0}\right| & \leqq(2(M+1))^{1 / n}, \\
1 / \Re\left|z_{0}\right| & \leqq 1, \\
\left|z_{0}\right| & \leqq \Re .
\end{aligned}
$$


This is false and the lemma is proved. ${ }^{2}$

We shall say that $\mathcal{F}$ has gaps of Hadamard type provided there is a positive number $\theta(\mathcal{F})=\theta$ and, for each $f \in \mathcal{F}$, a pair of sequences of positive integers $\left\{\lambda_{n}(f)\right\}_{n \geq 1},\left\{\mu_{n}(f)\right\}_{n \geq 1}$ such that (a) $\lambda_{n}(f) \leqq \mu_{n}(f)$, $n \geqq 1$, (b) $a_{v}(f) \neq 0$ implies $\lambda_{n}(f) \leqq \nu \leqq \mu_{n}(f)$ for some $n \geqq 1$, and (c) $\lambda_{n+1}(f) \geqq(1+\theta) \mu_{n}(f), n \geqq 1$.

THEOREM 1. Suppose $\mathcal{F}$ has radius of finite normality 1 and that $|z|=1$ is not a cut for $\mathcal{F}$. Then if $\mathcal{F}$ has gaps of Hadamard type, the subfamily of partial sums $\Sigma\left[\mu_{n}(f)\right]$ is overconvergent.

Proof. Let $D$ be a simply connected domain containing $|z|<1$ and the points of $|z|=1$ at which $\mathcal{F}$ is regular. It clearly suffices to assume that $1 \in D$ and to prove that $\Sigma\left[\mu_{n}(f)\right]$ converges uniformly to F on $|z-1| \leqq \alpha$ for some positive $\alpha$.

We choose a positive integer $p$ such that $p^{-1}<\theta$, set $z=1 / 2\left(w^{p}+w^{p+1}\right)$ $=\phi(w)$, and define the family of functions $G: g(w)=f(\phi(w))$ $=\sum_{\nu=0}^{\infty} a_{\nu}(f)(\phi(w))^{\nu}=\sum_{\nu=0}^{\infty} b_{\nu}(g) w^{\nu}$. From $\phi(1)=1 \in D$ and the fact that $|w| \leqq 1, w \neq 1$ implies $|\phi(w)|<1$, we infer the existence of a positive $\epsilon$ such that $G$ is finitely normal in $|w|<1+\epsilon$. Lemma 1 now shows that the subfamily of partial sums of $G$ which is determined by the family of sequences of integers $\left\{(p+1) \mu_{n}(f)\right\}$ converges uniformly to $G$ on $|w| \leqq 1+\frac{1}{2} \epsilon$. With this in mind we observe that the image of $|w| \leqq 1+\frac{1}{2} \epsilon$ under $z=\phi(w)$ necessarily contains a full disk about $z=1$. Denote this disk by $|z-1| \leqq \alpha(\alpha>0)$. Now, since $p^{-1}<\theta$, the gap condition on $\mathcal{F}$ implies that

$$
\sum_{p=0}^{\mu_{n}(f)} a_{\nu}(f)\left(\frac{w^{p}+w^{p+1}}{2}\right)^{\nu}=\sum_{\nu=0}^{(p+1) \mu_{n}(f)} b_{\nu}(g) w^{\nu}
$$

By our previous remarks the family on the left, i.e., $\Sigma\left[\mu_{n}(f)\right]$, converges uniformly to $\mathcal{F}$ on $|z-1| \leqq \alpha$. Theorem 2 is proved.

THEOREM 2. Let $F$ have radius of finite normality 1 and suppose that the subfamily $\Sigma\left[m_{n}(f)\right]$ of partial sums of $\mathcal{F}$ is overconvergent. Then every $f$ can be written as the sum of two power series,

$$
f(z)=g(z)+h(z),
$$

where the family $\{g(z)\}$ has gaps of Hadamard type and the family $\{h(z)\}$ has radius of finite normality greater than 1 .

PROof. It suffices to prove the existence of absolute constants $\rho, \theta$

${ }^{2}$ Essentially the same proof shows that $|z|=R$ is always a cut for the complete family of partial sums. This proof of that fact was pointed out to the writer by Mr. Donald Deckart of Rice University. 
and $N(\rho<1, \theta>0, N \geqq 1)$ such that the inequalities

$$
\left|a_{\nu}(f)\right|<\rho^{\nu}, \quad m_{n}(f)<\nu<(1+\theta) m_{n}(f), \quad n \geqq N,
$$

hold for every $f \in \mathcal{F}$.

Ostrowski's paper [1] contains a statement (p. 191, line 4) which in effect implies the validity of the inequalities (2) in the special case in which $\mathcal{F}$ reduces to a sequence of functions $f_{k}(z)=\sum_{\nu=0}^{\infty} a_{\nu}(k) z^{\nu}$ $(k=1,2,3, \cdots)$. One sees easily that under the hypotheses of Theorem 2, Ostrowski's reasoning leads, with only trivial changes, to the more general set of inequalities (2). For the details of this reasoning we refer the reader to Ostrowski's paper [1].

\section{REFERENCES}

1. A. Ostrowski, Über Potenzreihen die überkonvergente Abschnittsfolgen besitzen, Berliner Sitzungsberichte (1923) pp. 185-192.

2. - UÜber eine Eigenschaft gewisser Potenzreihen mit unendlich vielen verschwindenden Koeffizienten, Berliner Sitzungsberichte (1921) pp. 557-565.

3. J. Hadamard, Essai sur l'etude des fonctions donnees par leurs developpements de Taylor, J. Math. Pures Appl. (4) vol. 8 pp. 101-186.

4. P. Dienes, The Taylor series, New York, Dover Publications, 1957.

5. G. Johnson, Jr., Collective singularities of families of analytic functions, Proc. Amer. Math. Soc. vol. 7 (1956) pp. 653-655.

6. L. J. Mordell, On power series with the circle of convergence as a line of essential singularities, J. London Math. Soc. vol. 2 (1927) pp. 146-148.

University of Pennsylvania 